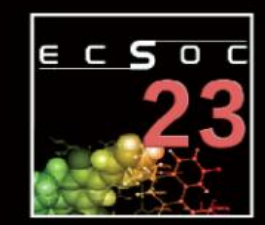

The 23rd International Electronic Conference on Synthetic Organic Chemistry

15 Nov-15 Dec 2019

chaired by Dr. Julio A. Seijas Vázquez

\title{
Formation of amino acid derived 2-thiohydantoins - An experimental and theoretical study
}

\section{Petar Stanić, ${ }^{1}$ Marija Živković, ${ }^{2}$ Zoran Marković, ${ }^{3}$ Dejan Milenković, ${ }^{3}$ Biljana $\breve{S} m i t^{3} *$}

\footnotetext{
${ }^{1}$ University of Kragujevac, Faculty of Science, Department of Chemistry, Radoja Domanovića 12, 34000 Kragujevac, Serbia ${ }^{2}$ University of Kragujevac, Faculty of Medical Sciences, Svetozara Markovića 69, 34000 Kragujevac, Serbia

${ }^{3}$ University of Kragujevac, Institute for Information Technologies Kragujevac, Jovana Cvijića bb, 34000 Kragujevac, Serbia *correspondence: biljana.smit@pmf.kg.ac.rs
}

\section{Abstract}

Hydantoins and thiohydantoins derived from amino acids are an interesting subset of compounds that have a wide range of chemical and biological properties. The chemistry of amino acid derived hydantoins does not require rigorous conditions and thus, their formation is plausible in physiological conditions. Hydantoins have been isolated from urine in cases where protein consumption is increased. This raises a question of chemical and biological implications of their presence and, accordingly, elucidation of mechanisms trough which they are formed gains importance.

The reaction of 2-thiohydantoin formation from corresponding amino acids was monitored by ${ }^{1} \mathrm{H}$ NMR spectroscopy and rate constants were determined. The mechanism of the reaction was examined using the M06-2X/6-311++G(d,p) theoretical model. The calculated rate constants of the reaction and the observed rate constants determined using NMR spectroscopy are in a good accordance. On the basis of the theoretical calculations, a different mechanism from the previously reported is proposed.

Keywords: thiohydantoin; amino acids; kinetics; mechanism; DFT 


\section{Introduction}

Hydantoins represent a large family of five-membered cyclic ureides with many interesting physical and chemical properties. [1] They encompass a wide set of structurally diverse compounds, which makes them very synthetically attractive. [2] They also exhibit many different biological activities, and many of them are frequently used in medicine and therapy as marketed drugs. [3]

With all things considered, we propose that a mechanistic, kinetic and theoretical study such as this one would prove beneficial to understanding the mechanisms of hydantoin formation, especially since, to our best knowledge, one has not been done yet. In this study, density functional theory (DFT) method is utilized to better understand the mechanisms of hydantoin formation and kinetics through which these processes are carried out. ${ }^{1} \mathrm{H}$ NMR spectroscopy monitoring is used as a useful tool in a searching for the most possible intermediates in the reaction. The theoretical results are discussed and compared with our experimental observations.

\section{Results and discussion}

One of the pioneering insights into the mechanisms of hydantoin formation is certainly Edman's work on peptide sequencing. [4] This method, called 'Edman degradation', involves one by one reaction of amino acids at the N-terminus with phenyl isothiocyanate, which produces the corresponding 2-thiohydantoin. Even though the methodology has received some critics and addendums [5,6], it still stands as an important landmark for, not only protein sequencing, but hydantoin chemistry.

In this study, we have monitored hydantoin formation in the reaction of several amino acid methyl esters and allyl isothiocyanate (allyl-NCS), as shown in Scheme 1.

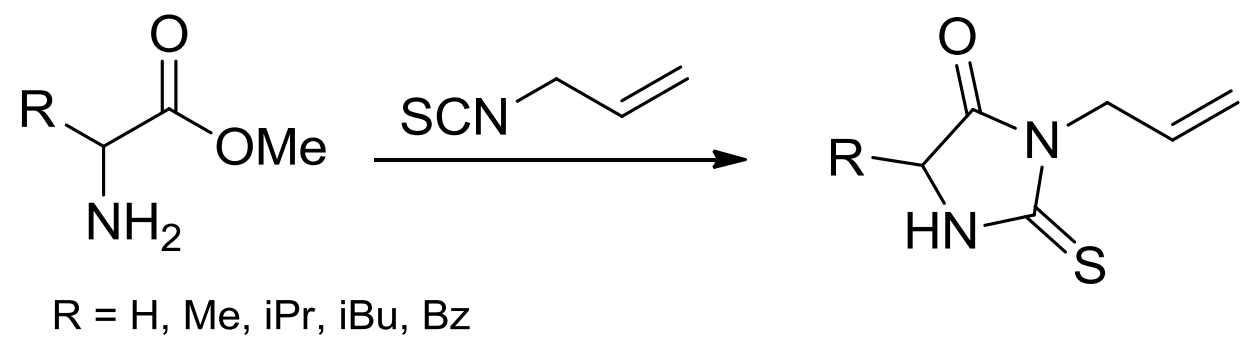

Scheme 1. Reaction of amino acid methyl esters with allyl-NCS 
The reactions were monitored by ${ }^{1} \mathrm{H}$ NMR spectroscopy and based on the spectral data, we propose a plausable two-step mechanism (Scheme 2), involving an allyl-NCS-amino acid intermedier, which undergoes cyclization to the corresponding 3-allyl-2-thiohydantoin derivative.

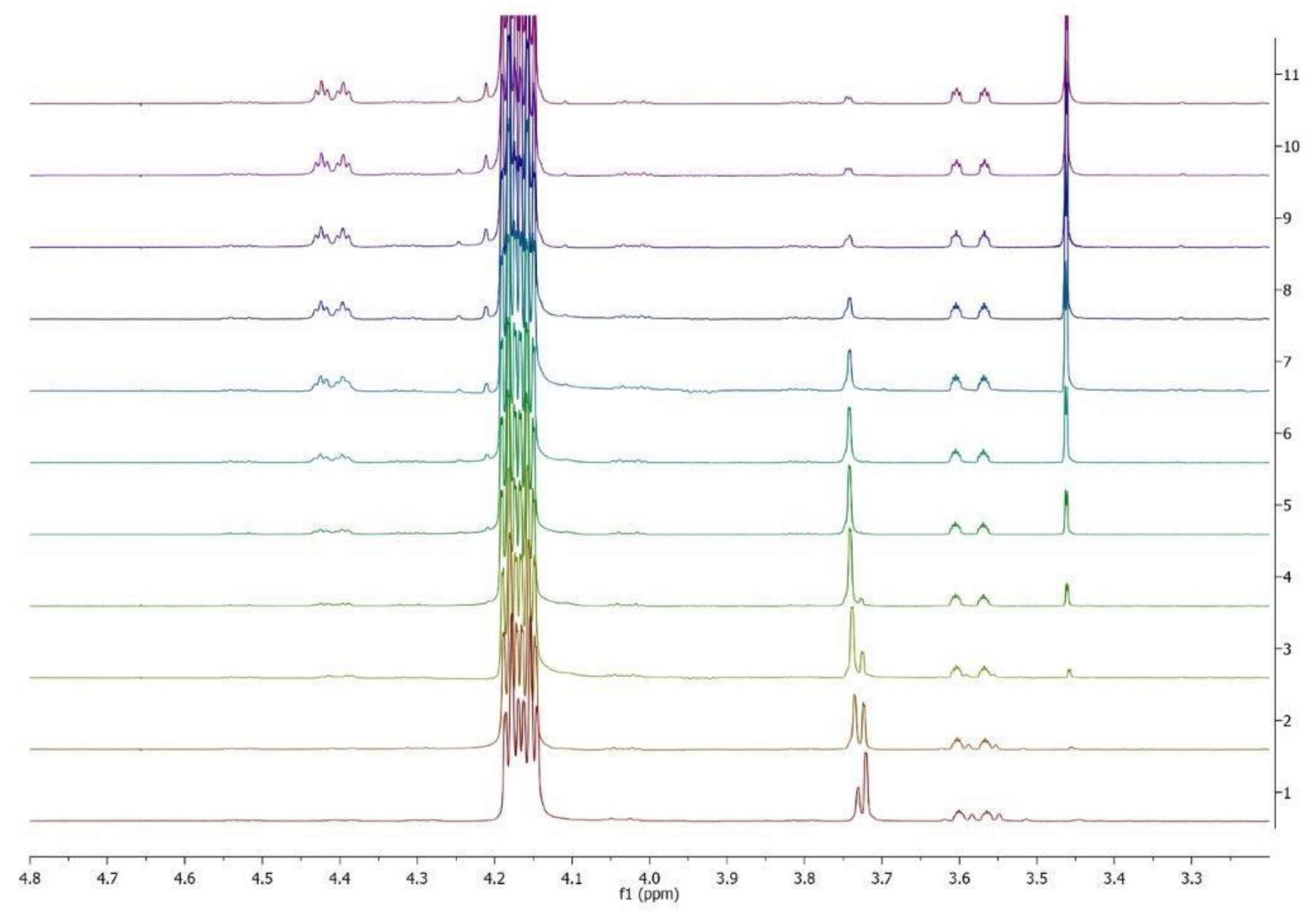

Figure 1. ${ }^{1} H$ NMR monitoring of the reaction between alanine methyl ester and allyl-NCS

In the spectra (Figure 1), we observed a singlet at $3.72 \mathrm{ppm}$ that belongs to the ester $\mathrm{OCH}_{3}$ protons of the amino acid methyl ester, which rapidly disappears. The singlet at $3.73 \mathrm{ppm}$, that belongs to the allyl-NCSamino acid intermedier, grows rapidly to a point, then slowly disappears right as the singlet that belongs to methanol protons at about $3.45 \mathrm{ppm}$ forms. All this points to a two step process in which the amino acid methyl ester reacts with allyl-NCS, forming the allyl-NCS amino acid intermedier, which readily undergoes cyclization in which methanol is released and the 3-allyl-2thiohydantoin derivative is formed. 


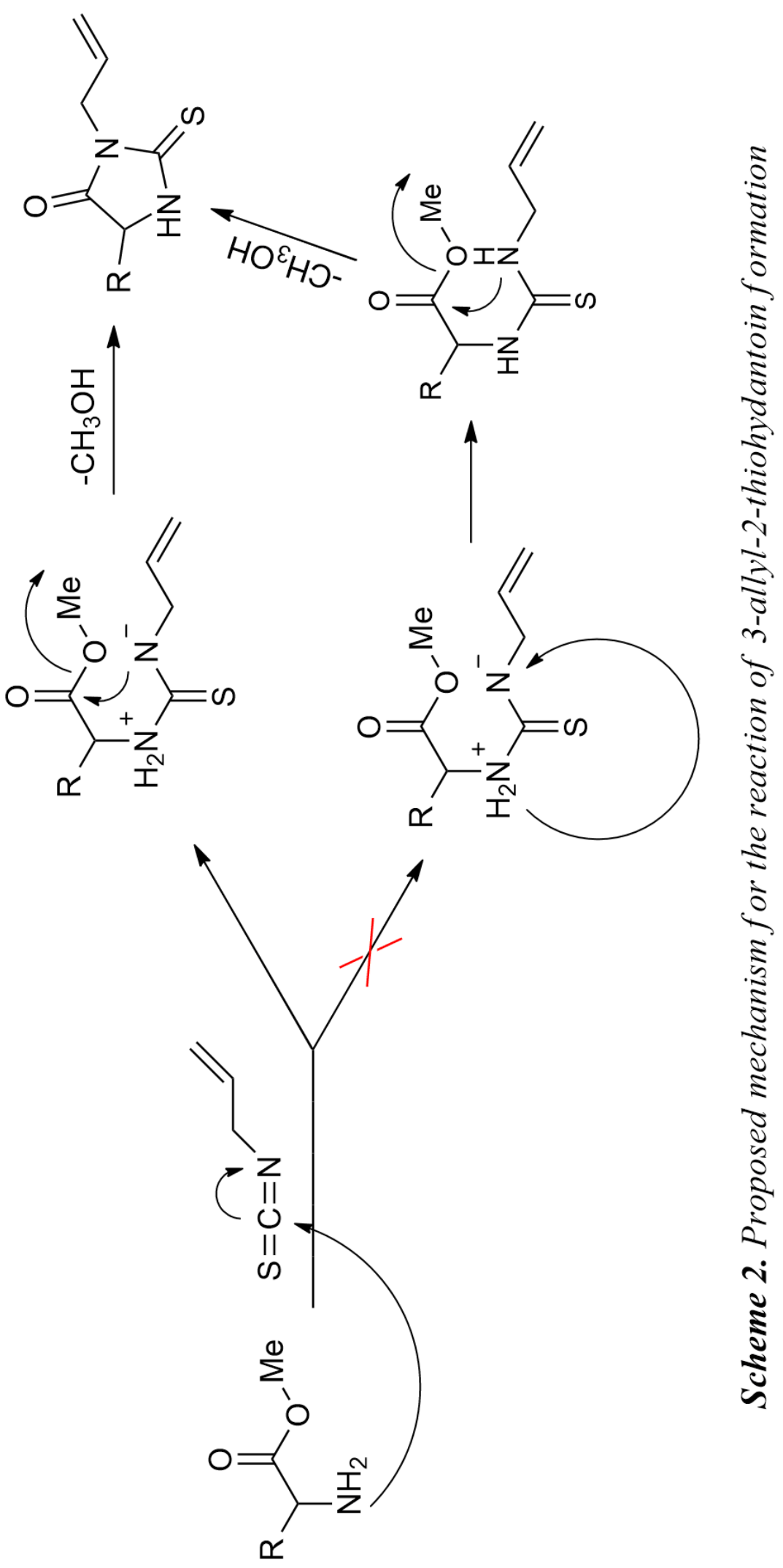


Peak integral values were used to calculate rate constants for both steps of the process. The changing ratio of integrals of the $\mathrm{OCH}_{3}$ proton peaks at 3.72 and $3.73 \mathrm{ppm}$ was used to calculate the rate constant of the formation of the allyl-NCS-amino acid intermedier, $k_{l}$. Formation of methanol, as seen from the slowly rising peak at $3.45 \mathrm{ppm}$ was used to calculate the rate constant of the cyclization step, $k_{2}$. Data is depicted in Table 1 . The reaction with glycine had the highest $k_{1}$ value, which indicates that glycine methyl ester undergoes nucleophilic adition to the allyl-NCS-amino acid reactive complex the fastest. Contrary, glycine had the lowest $k_{2}$ value, which means its reactive complex undergoes cyclization to the thiohydantion derivative the slowest. The highest $k_{2}$ value was for the reaction with phenylalanine.

Table 1. Rate constatnts for the two-step hydantoin formation process

\begin{tabular}{ccc}
\hline & $\begin{array}{c}\boldsymbol{k}_{\boldsymbol{l}} \\
\left(\mathrm{s}^{-1}\right)\end{array}$ & $\begin{array}{c}\boldsymbol{k}_{2} \\
\left(\mathrm{~s}^{-1}\right)\end{array}$ \\
\hline Gly-OMe + Allyl-NCS & $1.35 \times 10^{-3}$ & $4.18 \times 10^{-5}$ \\
Val-OMe + Allyl-NCS & $5.47 \times 10^{-4}$ & $5.44 \times 10^{-5}$ \\
Ala-OMe + Allyl-NCS & $7.50 \times 10^{-4}$ & $7.55 \times 10^{-5}$ \\
Leu-OMe + Allyl-NCS & $4.55 \times 10^{-4}$ & $6.67 \times 10^{-5}$ \\
Phe-OMe + Allyl-NCS & $5.28 \times 10^{-4}$ & $2.04 \times 10^{-4}$ \\
\hline
\end{tabular}

In order to gain a deeper insight into the reaction of the formation of amino acid derived 2-thiohydantoins, DFT was applied as well. The mechanism of the reaction between $\alpha$-amino acid methyl esters and allyl isothiocyanate (allyl-NCS) was examined using the M06-2X/6-311++G(d,p) theoretical model. Since there is no data in literature about the theoretical investigation of the reaction between allyl-NCS and $\alpha$ amino acid methyl esters, we used the mechanistic approach, applying the DFT method to examine this reaction. We found that the reaction of allyl-NCS and glycine methyl ester does not take place by the lower reaction pathway of proposed mechanism in Scheme 2. Namely, from Figure 2 it can be seen that the activation energy for this reaction pathway is $203.9 \mathrm{~kJ} \mathrm{~mol}^{-1}$, while the rate constant is $1.2 \times 10^{-23} \mathrm{~s}^{-1}$, indicating that reaction is not possible via this mechanism. On the basis of these facts, we considered that the key step, intramolecular cyclization, is realized through an attack of the internal nucleophile, the allylNCS nitrogen on the carbon of the $\mathrm{C}=\mathrm{O}$ group of the amino acid methyl ester, yielding the 3-allyl-2thiohydantoin products (Scheme 2, upper route). In this step, at the same time, the transfer of a hydrogen atom of the $\mathrm{NH}_{2}$ group to the oxygen of the $\mathrm{OCH}_{3}$ group occurs, following the formation of the cyclic reaction products. In all investigated cases, the attack of the internal nucleophile and transfer of the hydrogen atom/proton were the process of interest. 


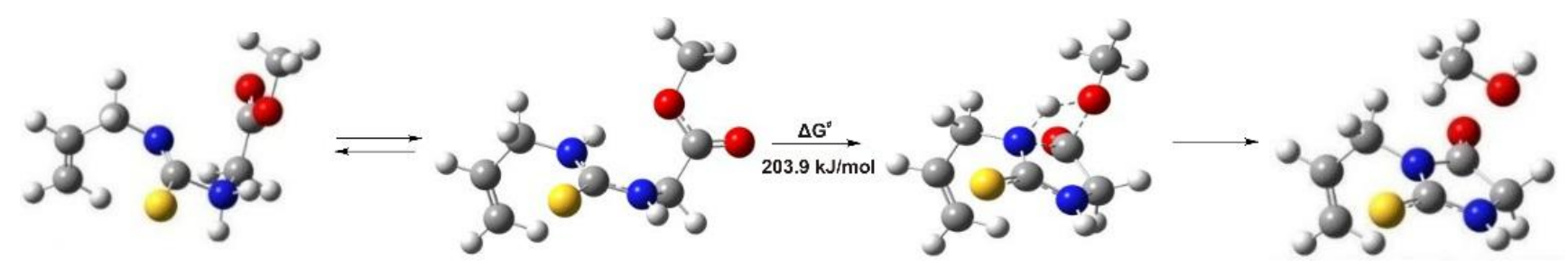

Figure 2. The reaction mechanism of the formation of a cyclic product between allyl-NCS and glycine methyl ester

The rate constants, activation energies and Gibbs free energy for all investigated reactions are presented in Table 1. The optimized geometries of RC, TS, and PC for the reactions of intramolecular cyclization and proton transfer are depicted in Table 1.

Table 1. The rate constants, activation energies and Gibbs free energy for the reaction of the formation cyclic products

\begin{tabular}{cccc}
\hline & $\begin{array}{c}\boldsymbol{k} \\
\left(\mathrm{s}^{-1}\right)\end{array}$ & $\begin{array}{c}\Delta \boldsymbol{G}^{\ddagger} \\
\left(\mathrm{kJ} \mathrm{mol}^{-1}\right)\end{array}$ & $\begin{array}{c}\Delta \boldsymbol{G}_{\boldsymbol{r}} \\
\left(\mathrm{kJ} \mathrm{mol}^{-1}\right)\end{array}$ \\
\hline Gly-OMe + Allyl-NCS & $4.42 \times 10^{-5}$ & 97.9 & -112.9 \\
Val-OMe + Allyl-NCS & $3.94 \times 10^{-5}$ & 98.2 & -106.9 \\
Ala-OMe + Allyl-NCS & $1.84 \times 10^{-4}$ & 94.3 & -116.3 \\
Leu-OMe + Allyl-NCS & $6.15 \times 10^{-5}$ & 97.1 & -107.1 \\
Phe-OMe + Allyl-NCS & $2.15 \times 10^{-4}$ & 93.9 & -117.0 \\
\hline
\end{tabular}

As can be seen from Table 2 and Figure 3, the activation energies and Gibbs free energies of the formation of cyclic products between phenylalanine methyl ester and allyl-NCS indicated that the reaction is kinetically and thermodynamically more favoured than other reactions, which is in agreement with the experimental results. 


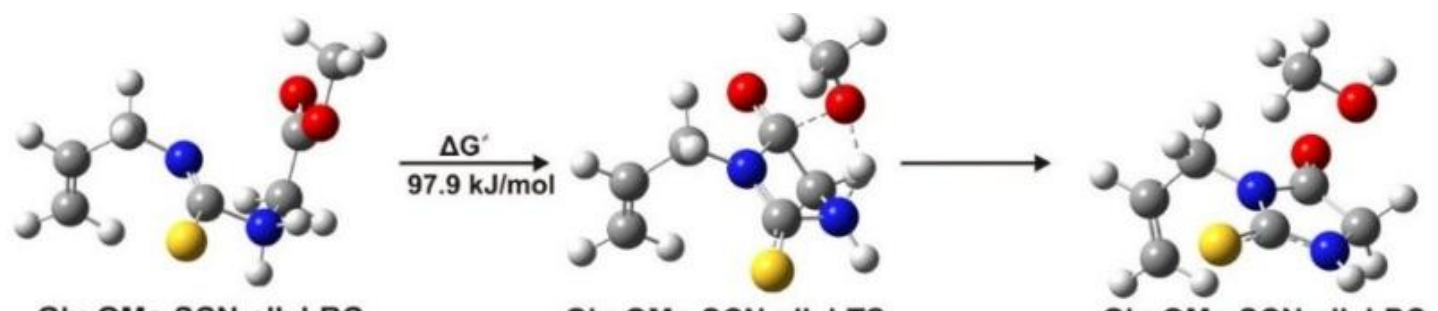

Gly-OMe-SCN-allyl-RC

Gly-OMe-SCN-allyl-TS

Gly-OMe-SCN-allyl-PC
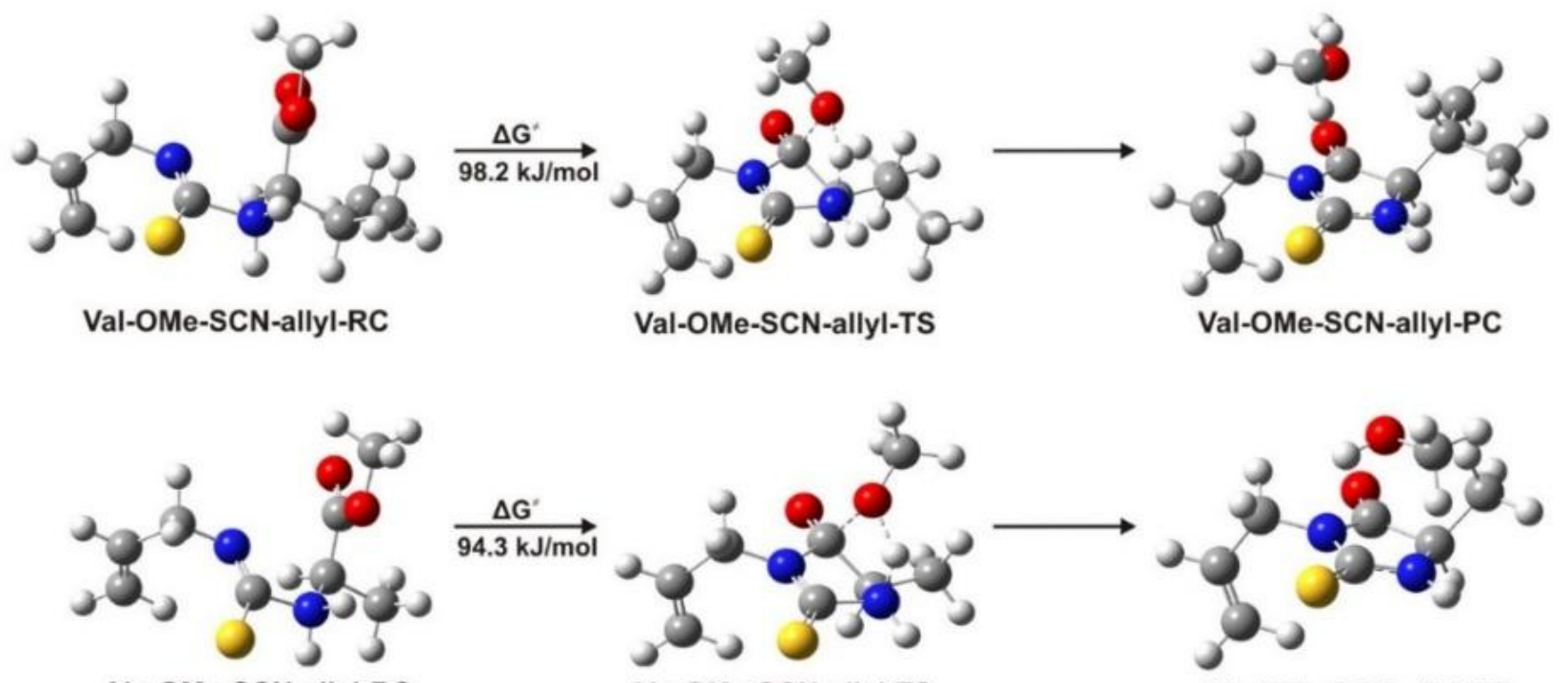

Ala-OMe-SCN-allyl-RC

Ala-OMe-SCN-allyl-TS

Ala-OMe-SCN-allyl-PC
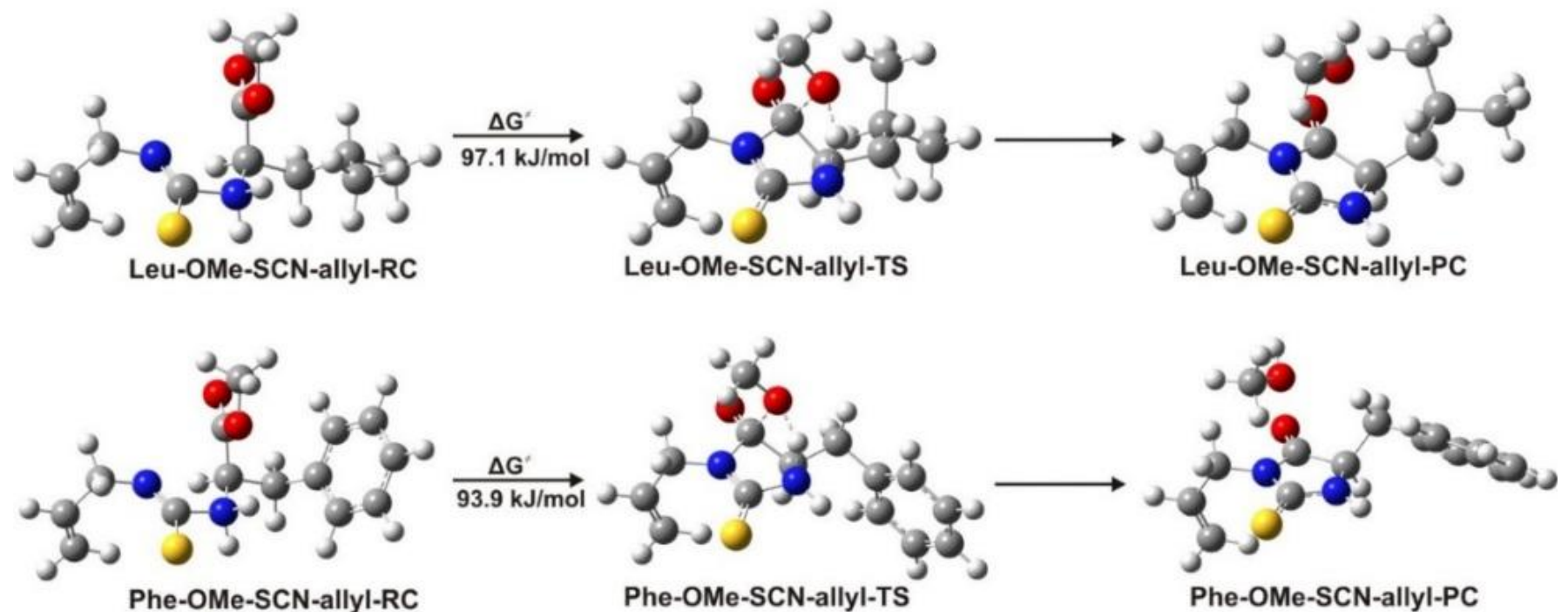

Figure 3. The optimized geometries of RC, TS, and PC for the reaction of intramolecular cyclization and proton transfer 


\section{Experimental}

\section{General}

All chemicals and reagents are commercially available and were used as received without further purification. Solvents were purified by distillation prior use. Anhydrous methanol was prepared by standard drying procedure. NMR spectra were recorded on a Varian Gemini 2000 NMR spectrometer in $\mathrm{D}_{2} \mathrm{O}$ or $\mathrm{CDCl}_{3}$ as solvents.

General procedure for the preparation of the amino acid methyl esters

Amino acid methyl esters were prepared according to a well known methanolic $\mathrm{HCl}$ method. $5 \mathrm{ml}$ of methanol was added to a round bottom flask and cooled to $0{ }^{\circ} \mathrm{C}$. Acetyl chloride $(2 \mathrm{ml})$ was added slowly to the stirred solution and then stirred for another 20 minutes at $0{ }^{\circ} \mathrm{C}$ to generate methanolic $\mathrm{HCl}$. $\mathrm{An}$ amino acid $(5 \mathrm{mmol})$ was added in one portion and the reaction was stirred overnight at room temperature. The solvent was removed in vacuo and solid amino acid methyl ester hydrochloride (yields ranging from 88 to $96 \%$ ) was used without further purification. Successful esterification was confirmed by ${ }^{1} \mathrm{H}$ NMR spectroscopy.

\section{Computational methods}

Geometry optimizations for all participants under investigation and frequency calculations have been carried out using a DFT method M06-2X combined with the 6-311++G(d,p) basis set. In order to encounter for the solvent effects, the reaction was modeled in chloroform $(\varepsilon=4.7113)$ by the CPCM polarizable continuum solvation model [7], as implemented in Gaussian 09 software package [8]. Geometries were fully optimized without imposing any restriction. Energy minimum structures contained no imaginary frequency, while transition states were characterized by one imaginary frequency.

The Intrinsic reaction coordinate (IRC) was used to verify that the imaginary frequency corresponds to the expected motion along the reaction coordinate in the case of the transition states. These calculations proved that each transition state (TS) connects two corresponding energy minima: reactant complex (RC) and product complex (PC). Natural bond orbital (NBO) analysis was performed for all participants in a simulated reaction between allyl isothiocyanate with selected compounds $[9,10]$. Transition state theory (TST) affords one of the simplest theoretical approaches for estimating the rate constants $(k)$, which 
requires only structural, energetic, and vibrational frequency information for reactants and transition states [11]. The main advantage of using conventional TST is that it requires very limited potential energy information (only on reactants and the transition state), which makes it practical for a wide range of chemical reactions. Despite its relative simplicity, this theory has been proven to be good enough to reproduce experimental rate constants [12].

The rate constant for the reaction between allyl isothiocyanate with $\alpha$-amino acid methyl esters was calculated using TST, implemented in TheRate program [13] and $1 \mathrm{M}$ standard state is calculated as follows:

$$
k=\frac{k_{\mathrm{B}} T}{h} \exp \left(\frac{-\Delta G^{\neq}}{R T}\right)
$$

where $k_{B}$ and $h$ stand for the Boltzmann and Planck constants, $\Delta G^{\neq}$is the free energy of activation, which is calculated as the difference in energies between transition states and reactants.

\section{Conclusions}

In summary, we have experimentally and theoretically examined thiohydantoin formation in the reactions of amino acid methyl esters with allyl isothiocyanate. By ${ }^{1} \mathrm{H}$ NMR monitoring of the reaction we proposed a two-step mechanism which involves a nucleophilic attach of the $\mathrm{NH}_{2}$ nitrogen of the amino acid on the NCS carbon of allyl isothiocynate in the first step. The second and key step, intramolecular cyclization, is realized through an attack of the internal nucleophile, the allyl-NCS nitrogen on the carbon of the $\mathrm{C}=\mathrm{O}$ group of the amino acid methyl ester, forming 3-allyl-2-thiohydantoin products. DFT calculations support

this. Rate constants have been calculated from the spectral data. Theoretical calculations are in good agreement with our experimental results.

\section{Acknowledgements}

The authors are grateful to the Ministry of Education, Science and Technological Development of the Republic of Serbia (Project numbers 172016, 172034 and 172036) for financial support.

\section{References}

1. E. Ware, Chem. Rev., 1950, 46, 403-470

2. L. Konnert, F. Lamaty, J. Martinez, E. Colacino, Chem. Rev., 2017, 117, 13757-13809 
3. S. Cho, S-H. Kim, D. Shin, Eur. J. Med. Chem., 2019, 164, 517-545

4. P. Edman, Acta Chem. Scand., 1950, 4, 283-293

5. L'. Drobnica, J. Augustin, Collect. Czech. Chem. Commun. 1965, 30, 99-104

6. K. Cejpek, J. Valušek, J. Velíšek, J. Agric. Food Chem., 2000, 48, 3560-3565

7. M. Cossi, N. Rega, G. Scalmani, V. Barone, J. Comput. Chem., 2003, 24, 669-681

8. M. J. Frisch, G. W. Trucks, H. B. Schlegel, G. E. Scuseria, M. A. Robb, J. R. Cheeseman, G. Scalmani, V. Barone, B. Mennucci, G. A. Petersson, H. Nakatsuji, M. Caricato, X. Li, H. P. Hratchian, A. F. Izmaylov, J. Bloino, G. Zheng, J. L. Sonnenberg, M. Hada, M. Ehara, K. Toyota, R. Fukuda, J. Hasegawa, M. Ishida, T. Nakajima, Y. Honda, O. Kitao, H. Nakai, T. Vreven, J. A. Montgomery, J. E. Peralta, F. Ogliaro, M. Bearpark, J. J. Heyd, E. Brothers, K. N. Kudin, V. N. Staroverov, R. Kobayashi, J. Normand, K. Raghavachari, A. Rendell, J. C. Burant, S. S. Iyengar, J. Tomasi, M. Cossi, N. Rega, J. M. Millam, M. Klene, J. E. Knox, J. B. Cross, V. Bakken, C. Adamo, J. Jaramillo, R. Gomperts, R. E. Stratmann, O. Yazyev, A. J. Austin, R. Cammi, C. Pomelli, J. W. Ochterski, R. L. Martin, K. Morokuma, V. G. Zakrzewski, G. A. Voth, P. Salvador, J. J. Dannenberg, S. Dapprich, A. D. Daniels, Ö. Farkas, J. B. Foresman, J. V. Ortiz, J. Cioslowski, D. J. Fox, Gaussian 09, Revision C.01, Gaussian, Inc., Wallingford CT, 2009

9. J. E. Carpenter, F. Weinhold, J. Mol. Struct. THEOCHEM., 1988, 169, 41-62

10. E. D. Glendening, J. K. Badenhoop, A. E. Reed, J. E.Carpenter, J. A. Bohmann, C. M. Morales, F. Weinhold, NBO 5.9, 2009

11. A. Galano, M. Francisco Marquez, A. Pérez-González, Chem. Res. Toxicol., 2014, 27, 904-918

12. A. Galano, J.R. Alvarez-Idaboy, J. Comput. Chem., 2013, 34, 2430-2445

13. W. T. Duncan, R. L. Bell, T. N. Truong, J. Comp. Chem., 1998, 19, 1039-1052 